\title{
OPTIMIZED BIOMETRIC SYSTEM BASED ON COMBINATION OF FACE IMAGES AND LOG TRANSFORMATION
}

\author{
Sateesh Kumar $\mathrm{H} \mathrm{C}^{1}$ Raja K B ${ }^{2}$ and Venugopal $\mathrm{KR}^{3}$ \\ ${ }^{1}$ Department of Electronics and Communication Engineering, \\ Sai Vidya Institute of Technology, Bangalore, India \\ ${ }^{2}$ Department of Electronics and Communication Engineering, \\ University Visvesvaraya College of Engineering, Bangalore, India \\ ${ }^{3}$ Principal, University Visvesvaraya College of Engineering, Bangalore, India
}

\begin{abstract}
The biometrics are used to identify a person effectively. In this paper, we propose optimised Face recognition system based on log transformation and combination of face image features vectors. The face images are preprocessed using Gaussian filter to enhance the quality of an image. The log transformation is applied on enhanced image to generate features. The feature vectors of many images of a single person image are converted into single vector using average arithmetic addition. The Euclidian distance $(E D)$ is used to compare test image feature vector with database feature vectors to identify a person. It is experimented that, the performance of proposed algorithm is better compared to existing algorithms.
\end{abstract}

\section{KEYWORDS}

Biometric, Face recognition, log Transformation, ED, Fusion, Gaussian filter

\section{INTRODUCTION}

Biometrics is the measurement and analysis of behavioural and physiological trait characteristics of a person. It is used to identify a person to utilise electronic gadgets and entry in to restricted areas through smart gates or doors. The conventional human authentication methods used are smart cards, passwords, Personnel identification number (PIN) etc. The disadvantages are (i) Passwords are hard to remember (ii) PIN and smart cards can be stolen or lost. Biometrics is the alternate to conventional methods of authentication as the traits of biometrics are attached to human body parts and based on the behaviour of person. The biometric system has three divisions viz., (i) enrolment division (ii) test division and (iii) matching division. In enrolment division, the database images are loaded preprocessed and features are extracted. In test division, the test images are loaded, preprocessed and features are extracted. The matching division has classifiers to classify images inside the database. 
Signal \& Image Processing : An International Journal (SIPIJ) Vol.7, No.2, April 2016

The biometrics are broadly divided into two categories viz., (i)Physiological biometric traits which are related to parts of human body and the characteristics are constant in lifetime and (ii)behavioural biometric traits, that are based on behaviour of a person like walking style, writing style, speaking accent etc., which vary in lifetime. In our research work, face images are used to identify a person. The advantage of this trait is, images can be captured without the cooperation of a person. The challenges in face recognition are (i) Orientation of face images, (ii) Illumination variations (iii) Accessories of face images and (iv) Expressions.

The applications of biometrics are (i) Identification of a person in crowded places like bus stand, railway station, airport etc., using face recognition system. (ii)The fingerprint identification is used to identify criminals in the scenario of crime (iii) The biometrics are used in cloud computing (iv) Used in secured communication(v)Used in IOT (vi)Used in National Identification card (AADHAR) to create national database (vii)Bank transactions (viii) Property registration (ix)to protect Intellectual property.

Contribution: In this paper, the novel concept of converting feature vectors of many images with illumination variations, orientation variation etc., of a single person into one feature vector per person. The Gaussian filer and log transformations are used to enhance quality of images. The features are extracted using log transformations.

Organisation: Survey of recent research is discussed section 2. Section 3 presents proposed model. Proposed Algorithm is described in section 4. Performance Analysis is explained in the section 5. Conclusion is given in Section 6.

\section{LITERATURE SURVEY}

In this section, the literature review of existing biometric algorithms is discussed. Abhijith Punnappurath and Ambasamudram Narayanan Rajagopalan [1] proposed a face recognition technique under non uniform blur, Illumination and pose. The blurred images are modelled as a convex set. Illumination variations are handled by exploiting the fact non uniform blurred and changed illumination image forms Biconvex set. The disadvantage of this technique is large changes in the face and significant occlusions cannot be handled. Yong You et al.,[2] presented high accuracy Face Recognition technique with lower complexity for image with variable illumination, pose and facial expressions. This is achieved by first exploiting the original training samples to synthesise virtual training samples, which reflects the possible variations in the face. A theorem is used to fix upper bound for number of useful training samples.

Chia -Po Wei et al.,[3] proposed face recognition when the data is corrupted due to occlusion or disguise. This problem is addressed by low rank matrix decomposition. This gives additional discriminating ability as it enforces bases learned for different classes are independent. Muwei Jian and Kin-Man Lam[4] proposed a novel approach for face recognition and verification of low resolution of images based on singular value decomposition. This technique is based on the fact that low resolution (LR) and high resolution (HR) images have linear relation. Based on LR-HR pairs, the mapping functions for interpolating the two matrices in SVD representation for reconstruction of high resolution images is done accurately. Changxing Ding et al.,[5] proposed robust face recognition technique to variation in the pose, expression and illumination. This method extracts multi directional, multi level dual-cross patterns. Difference in illumination is 
Signal \& Image Processing : An International Journal (SIPIJ) Vol.7, No.2, April 2016

addressed by taking first derivative of Gaussian operator. It increases the robustness in pose variation and expression variation but it doubles the cost of local binary pattern.

Yi Wen Wang and Shutao Li[6] proposed a rapid face recognition based on the brain computer interface. It uses combination of electro encephalography signal along with regular face recognition technique. In neuroscience face images stands differently, it produces large amplitude of face specific components in EEG other than natural images. Leyuan Fang et al.,[7] proposed a Gabor features based face recognition technique which exploits the correlated Gabor features using multitask adoptive sparse representation model. This algorithm gives better representation, as selected atoms for the Gabor features are varied with in each class. Jiwen Lu et al.,[8] proposed a joint feature learning technique for face recognition. In this method different regions of the face are considered will have different physical characteristics. By jointly learning multiple and related sparse features for different face regions, more position specific discriminative information is extracted for face representation.

Hamdi Dibeklioglu et al.,[9] proposed age estimation method by capturing face images. Age is estimated by studying dynamic facial features by analyzing frequently changing facial expressions. The spontaneous and posed smiles have different and distinct dynamics concept outperform over other technique.Rosario Campomanes-Alvarez et al.,[10] proposed a method for identifying a person by overlaying skulls with the images of missing person.This is done by modelling impression related to the facial soft tissue depth between corresponding pairs of cranial and facial landmarks.

Shenghua Gao et al.,[11] proposed a supervised auto encoder and used it to build deep neural network architecture for extracting robust features for single sample per person representation. Here face images are enforced with variants to be mapped with canonical face of the person and also enforcing features corresponding to same person which are similar. Yiwen Wang et al.,[12] proposed a close loop face retrieval which combines existing face recognition with EEG signals. In this method random face images are taken and outputs the ranking of all of the images in the data base according to their similarities to the target individual. At each iteration event related potentials are taken. Xiaochun Cao et al.,[13] proposed a face clustering technique to cluster videos. The constrained multi view video face clustering under a unified graph based method is used. The constrained sparse subspace representation is forced to explore unknown relationships. In the constrained spectral clustering, the constraints are used to guide for learning more reasonable new representations.

Xudong Jiang and Jian Lai[14] proposed a sparse and dense-hybrid representation based face recognition which uses low rank dictionary decomposition/learning. In this method every sample uses its class specific component to compete against the others collaboratively with the non-class specific components of all the samples. Priyanka V. Bankar and Anjali C. Pise[15] proposed a face recognition method using GABOR and LBP. This method encodes the discriminative features by combining both colour and text information. To make full use of colour and texture information, the opponent colour texture features are used. Muhammad Uzair et al.,[16] proposed a hyper spectral face recognition. It uses a spatial- spectral covariance for band fusion and partial least square regression for classification. Hyper spectral face recognition is an image set classification problem and performance is evaluated using seven state of art image set classification techniques. Samiksha Agrawal and Pallavi Khatri [17] proposed a high speed accurate face recognition algorithm based on Viola and Jones algorithm and principle component 
Signal \& Image Processing : An International Journal (SIPIJ) Vol.7, No.2, April 2016

analysis. The principle component analysis uses eigen phases and reconstruction of phase is possible with small amount of information. Xiao Shuang Shi et al.,[18] proposed a method to reduce the influence of grosses like variations in lighting, facial expressions and occlusions to improve the robustness of Face recognition system. The two dimensional whitening reconstruction reduces the pixel redundancy of internal image and it maintains important intrinsic features this improve the robustness of PCA methods on classification and clustering, especially for faces with severe illumination changes.

\section{PROPOSED MODEL}

The novel technique of converting many image feature vectors of single person into one image feature vector is introduced in the proposed algorithm and block diagram is shown in the figure 1. The Gaussian filter, Log Transformation and fusion technique are used to optimize the algorithms.

\subsection{Face Image Databases:}

The proposed algorithm is tested using some of the universally available databases such as JAFFE, YALE, Indian male, Indian females.

1. JAFFE Database [19] (Japanese Female Facial Expressions database):It consists of 213 Images. It has 7 facial expressions such as neutral, angry, happiness, disgust, sad, fear and disgust. Out of seven facial expressions 6 are basic facial expressions and one is neutral expression. These are the images taken from 10 Japanese female models. The database is created by considering 6 images per person, there are 42 database images. JAFFE database images are as shown in figure 2

2. YALE Database [20]: It consists of 165 gray scale images in GIF format of 15 persons.. 11 images were taken from each person. It contain Different facial expressions like Centre light, With glasses, happy, left light, with no glasses, normal, right-light, sad, sleepy surprised and wink. The database is created by taking images of 10 persons with 6 samples per person. Figure 3 shows Yale database images.

3. Indian male database [21]: Indian male face database has twenty persons with approximately eleven images per person. The images were taken in homogeneous background with an upright and frontal position. The eleven different images include facial orientations such as looking front, looking left, looking right, looking up, looking up towards left, looking up towards right, looking down, with emotions neutral, smile, laughter, sad/disgust. The database is formed by taking images of 15 persons with 6 images per person. Samples of Indian male face images of a person are shown in fig. 4.

4. Database of Indian female [21]: The face database of Indian females has twenty two persons with nearly eleven images per person. Pose and expressions variations are same as Indian male face database. The database is formed by considering first 17 persons and 6 images of each person. Total images in the database are 102. Out of database persons are 5. Hence total images outside the database Figure 5 shows Indian female face image samples of a person. 
Signal \& Image Processing : An International Journal (SIPIJ) Vol.7, No.2, April 2016

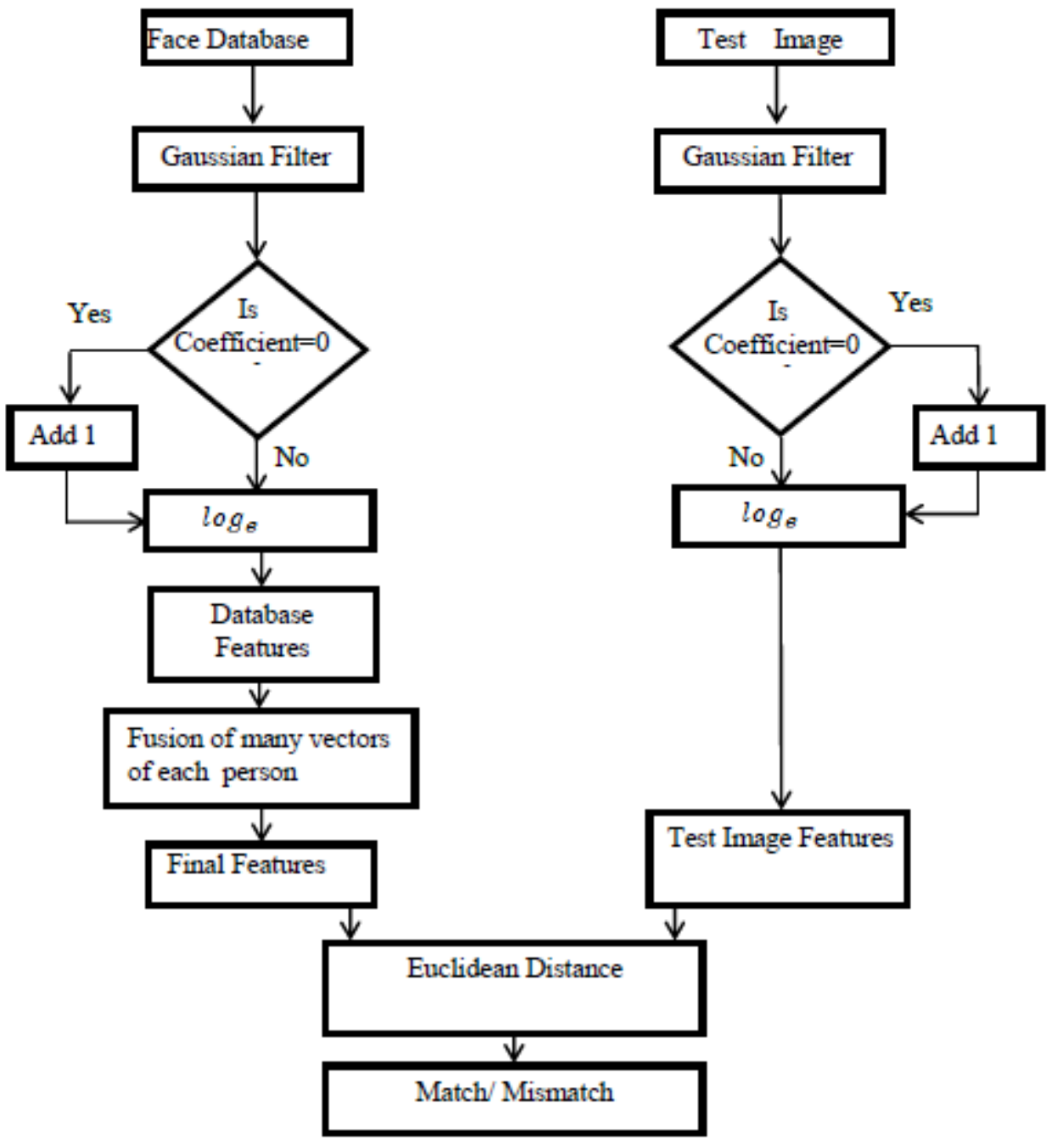

Figure 1. Block diagram of proposed Face Recognition using Natural logarithm and vector compression

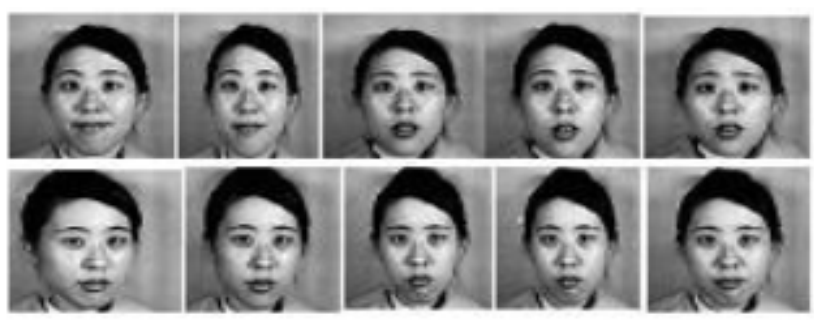

Figure 2. Samples of JAFFE face images of a person 
Signal \& Image Processing : An International Journal (SIPIJ) Vol.7, No.2, April 2016
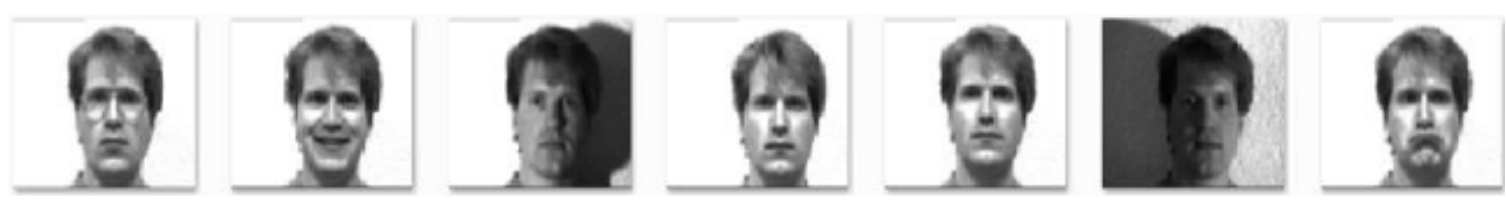

Figure 3. YALE face image samples of a person

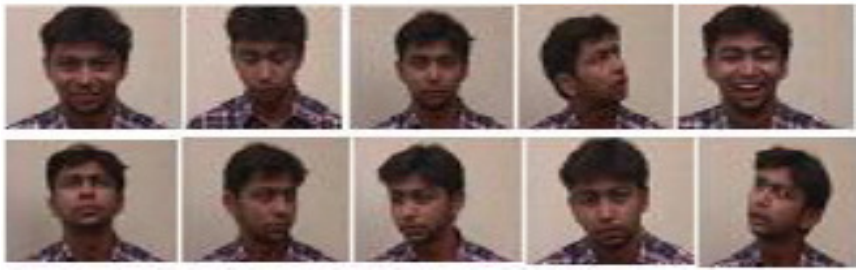

Figure 4. face image Samples a person of Indian male

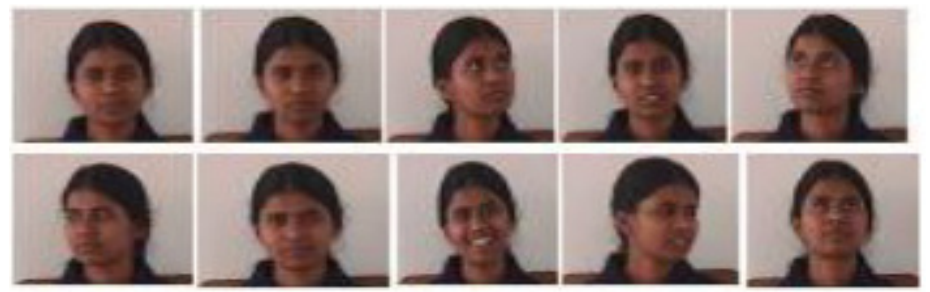

Figure 5. Indian female face image Samples of a person

\subsection{Gaussian Filter [22]:}

The filter is used to transform the image to smoothened image by removing high frequency edges. The basic equation for two dimensional Gaussian filter is given in equation (1).

$$
g(x, y)=\frac{1}{2 \pi \sigma^{2}} e^{-\frac{x^{2}+y^{2}}{2 \sigma^{2}}}
$$

Where, $\mathrm{x}$ is the distance from the origin in the horizontal axis

$\mathrm{y}$ is the distance from the origin in the vertical axis.

$\sigma$ is the standard deviation of the Gaussian distribution.

The Gaussian mask filter of $3 \times 3$ is derived from equation (1) to obtain mask given in equation (2).

Gaussian Mask $=\frac{1}{16}\left[\begin{array}{lll}1 & 2 & 1 \\ 2 & 4 & 2 \\ 1 & 2 & 1\end{array}\right]$ 
Signal \& Image Processing : An International Journal (SIPIJ) Vol.7, No.2, April 2016

The Gaussian filter is obtained by multiplying Gaussian mask with $3 \times 3$ sub-matrix of an image as given in equation 3 .

Gaussian Filter $=\frac{1}{16}\left[\begin{array}{lll}1 & 2 & 1 \\ 2 & 4 & 2 \\ 1 & 2 & 1\end{array}\right] *\left[\begin{array}{lll}a_{33} & a_{32} & a_{31} \\ a_{23} & a_{22} & a_{21} \\ a_{13} & a_{12} & a_{11}\end{array}\right]$

Where, $a_{11}$ to $a_{33}$ are $3 \times 3$ sub-matrix pixel values of an image.

\section{Gaussian Filter}

$$
\begin{aligned}
& =\frac{1}{16}\left[\left(a_{33}+2 a_{32}+a_{31}\right)+\left(2 a_{23}+4 a_{22}+2 a_{21}\right)+\left(a_{13}+2 a_{12}\right.\right. \\
& \left.\left.+a_{11}\right)\right] \\
& =\frac{1}{16}\left[\left(a_{33}+a_{31}+a_{13}+a_{11}\right)+2\left(a_{32}+a_{23}+a_{21}+a_{12}\right)+4 a_{22}\right]
\end{aligned}
$$

\subsection{Log Transformation}

It is a spatial domain technique which operates directly on the pixel intensities. The low and high Intensity values are converted into medium intensity values i.e., intensity values are distributed uniformly in an image. The very low intensity values are increased to moderate values and high intensity values are reduced to moderate values using log transformation. The gray level intensity values of transformation is given by the equation (5)

$$
\mathrm{s}=\mathrm{Clog}(1+\mathrm{r})
$$

where, $\mathrm{c}$ is constant, $\mathrm{r}=$ Intensity value of original Image

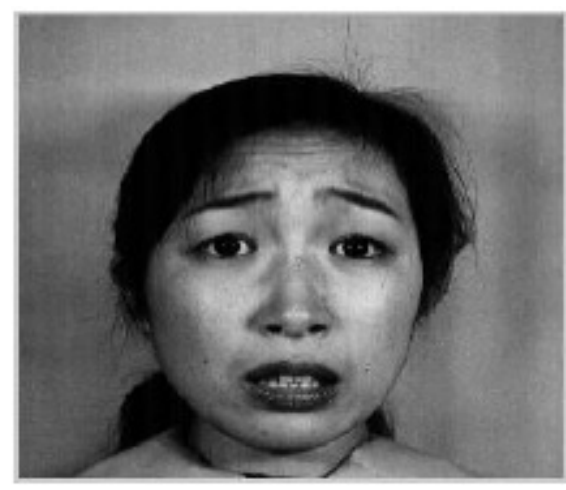

a) Original image

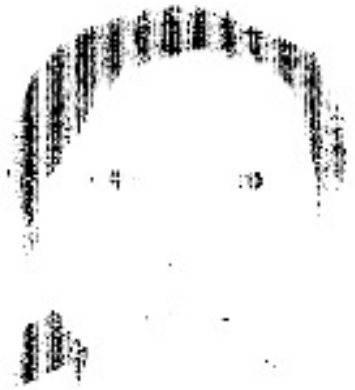

(b) log image

Figure 6. Logarithmic transformation of an image

The log transformation of an image is as shown in fig 6. The log transformation is applied on all database images to obtain matrix of log coefficients. The matrix of each image is converted into column vector. The database column vectors of log coefficients for all images are derived in the database features. 
Signal \& Image Processing : An International Journal (SIPIJ) Vol.7, No.2, April 2016

\subsection{Fusion of Vector:}

It is the process of converting many feature vectors of single person into one vector using average arithmetic addition of corresponding coefficients. The algorithm is optimized since only one vector for each person.

Example:

Let seven images per person and the corresponding features vectors are say $\mathrm{V}_{1}, \mathrm{~V}_{2}, \mathrm{~V}_{3}, \mathrm{~V}_{4}, \mathrm{~V}_{5}, \mathrm{~V}_{6}$ and $\mathrm{V}_{7}$. The image size is considered as $256 * 256$ ie., number of features for each image are 65536. The feature elements in each vector are given in equation (6)

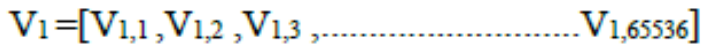

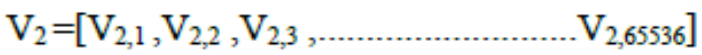

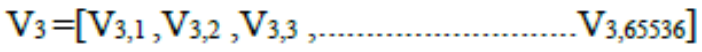

$$
\begin{aligned}
& \mathrm{V}_{4}=\left[\mathrm{V}_{4,1}, \mathrm{~V}_{4,2}, \mathrm{~V}_{4,3}, \ldots \ldots \ldots \ldots \ldots \ldots \ldots \ldots . . . . . . . . . . .65536\right]
\end{aligned}
$$

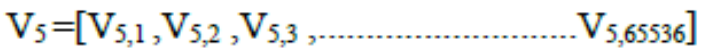

$$
\begin{aligned}
& \mathrm{V}_{6}=\left[\mathrm{V}_{6,1}, \mathrm{~V}_{6,2}, \mathrm{~V}_{6,3}, \ldots \ldots \ldots \ldots \ldots \ldots \ldots \ldots . . . . . . . . . .65536\right]
\end{aligned}
$$

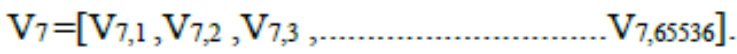

The seven feature vectors are converted into single vector (V) using fusion technique given in equation (7)

$$
\mathrm{V}=\left[\mathrm{V}_{\mathrm{F} 1}, \mathrm{~V}_{\mathrm{F} 2}, \mathrm{~V}_{\mathrm{F} 3}\right.
$$

$$
\text { where } \begin{aligned}
\mathrm{V}_{\mathrm{F} 1}= & \frac{\sum_{i=1}^{7} V_{i, 1}}{7} \\
\mathrm{~V}_{\mathrm{F} 2}= & \frac{\sum_{i=1}^{7} V_{i, 2}}{7} \\
\mathrm{~V}_{\mathrm{F} 3}= & \frac{\sum_{i=1}^{7} V_{i, 8}}{7} \\
& \cdot \\
\mathrm{V}_{\mathrm{F} 65536}= & \frac{\sum_{i=1}^{7} V_{i, 65586}}{7}
\end{aligned}
$$

Many feature vectors of single person is converted into one feature vector per person. The advantage is reduction in computation time, which will optimize the algorithm. 
Signal \& Image Processing : An International Journal (SIPIJ) Vol.7, No.2, April 2016

\subsection{Euclidian Distance:}

The Euclidian distance is used as matching metric to compare two feature templates of face images. Equation (8) gives Euclidian distance equation.

$$
\mathrm{d} 1(\mathrm{p}, \mathrm{q})=\sqrt{\sum_{\mathrm{i}=1}^{\mathrm{M}}\left(p_{i}-q_{i}\right)^{2}}
$$

Where, $M=$ The total number of feature coefficiants.

$$
\begin{aligned}
& p_{i}=\text { Face image's } i^{\text {th }} \text { feature value in the database } \\
& q_{i}=\text { Test image's } i^{\text {th }} \text { feature value. }
\end{aligned}
$$

\section{ALGORITHM}

In this section problem definition, objectives and algorithm are discussed and the proposed algorithm for face recognition is given in table 1.

problem definition: Optimised face recognition Algorithm is developed using log transformation and feature vector fusion to identify a person efficiently.

Table 1: Algorithm of proposed method

Input :Face database, Test face image.

Output: Recognition of face image.

1. The face database is created.

2. Quality of face image is enhanced using Gaussian filter.

3. The log transformation is applied on enhanced face images to extract the features.

4. The feature matrix of each image is converted into column vector.

5. The many feature column vectors corresponding to each face image of single person are converted into one feature column vector using fusion

6. step 2 to 4 are repeated for test image.

7. The ED is used to compare final features of database and test images to identify person. 
Objectives are-

i) To recognise the persons efficiently

ii) To increase recognition rate.

iii) To decrease error rates such as FRR, FAR and EER

In the proposed concept of converting many images of single person into one image per person using average arithmetic addition is introduced in the proposed algorithm.

\section{Performance Analysis}

In this section, the definitions of performance parameters, variations of performance parameters with threshold and comparison of proposed method with existing methods are discussed.

\subsection{Definition of performance parameters.}

\subsubsection{True Success Rate or Correct Recognition Rate (TSR or CRR):}

It is defined as the number of test images correctly matched with database images to the total number of persons in the database as given in equation (9)

$T S R=\frac{\text { No.of persons correctly mathched in the database }}{\text { Totalno.of persons in databse }}$

\subsubsection{False Rejection Rate (FRR):}

It is defined as the ratio of number of guanine test persons rejected to the total number of persons in the database as given in equation (10)

$$
F R R=\frac{\text { No.of correct persons rejected }}{\text { Total no.of persons in databse }}
$$

\subsubsection{False Acceptance Rate (FAR):}

It is the number of imposter persons accepted to the total number of persons in the outside the database as given in equation (11)

$$
F A R=\frac{\text { No.of persons accepted from out of database }}{\text { Total no.of persons in databse }} .
$$

\subsubsection{Equal Error Rate (EER):}

It is an optimum error of an Algorithm. The EER is the Intersection of FAR and FRR. The EER value must be low for better performance of an algorithm. 
Signal \& Image Processing : An International Journal (SIPIJ) Vol.7, No.2, April 2016

\subsection{Performance parameter variations:-}

In this section, The threshold values are varied and performance parameter values are noted for different face image databases such as Indian Female, Jaffe, Yale and Indian male.

\subsubsection{Parameter variation with Jaffe Face Database:-}

seven persons with six samples per person are used to create face database by considering seven persons with six samples per person. ie., forty two image samples in the database. The values of the FAR are computed by considering three persons as outside the database. The variations of FRR, FAR and TSR with threshold are shown in fig. 7

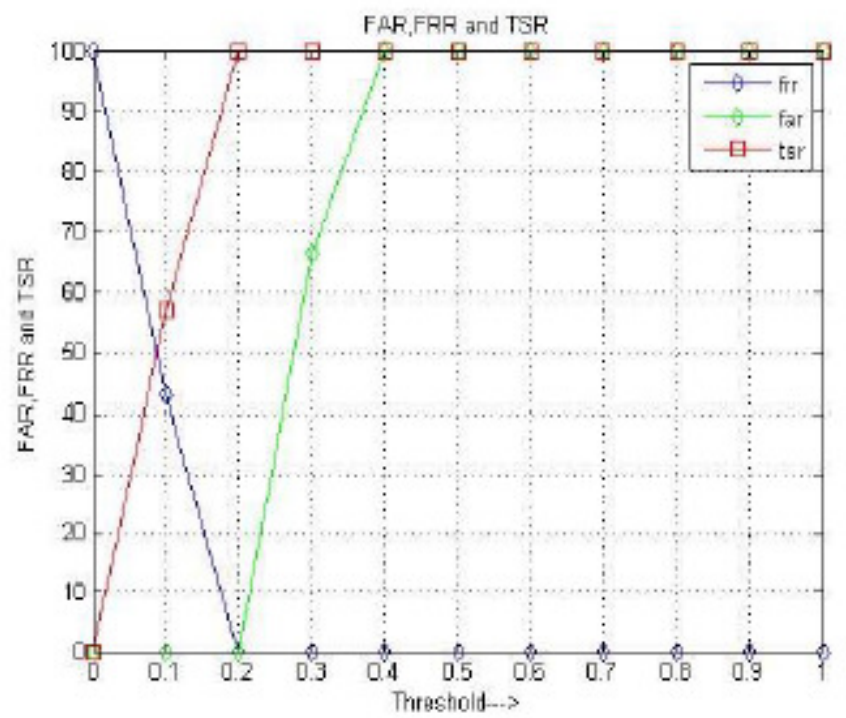

Figure 7. JAFFE database Performance parameters variations with threshold.

FAR and TSR values increases with threshold values, where as the FRR decreases with threshold values. The value of EER is zero and corresponding TSR value is one hundred percent The performance values are better since the variations in the image are less.

\subsubsection{Parameter variation with Yale Face Database:-}

The face database is formed by considering ten persons with six samples per person. ie., sixty image samples in the database. The value of the FAR is computed by considering five persons as outside the database. The variations of FRR, FAR and TSR with threshold are shown in fig. 8

The values of FAR and TSR increases with threshold values, where as the FRR decreases with threshold values. The value of EER is thirty two percent and corresponding TSR value is seventy percent. The performance values are poor since the variation of illuminations in images are more. 
Signal \& Image Processing : An International Journal (SIPIJ) Vol.7, No.2, April 2016

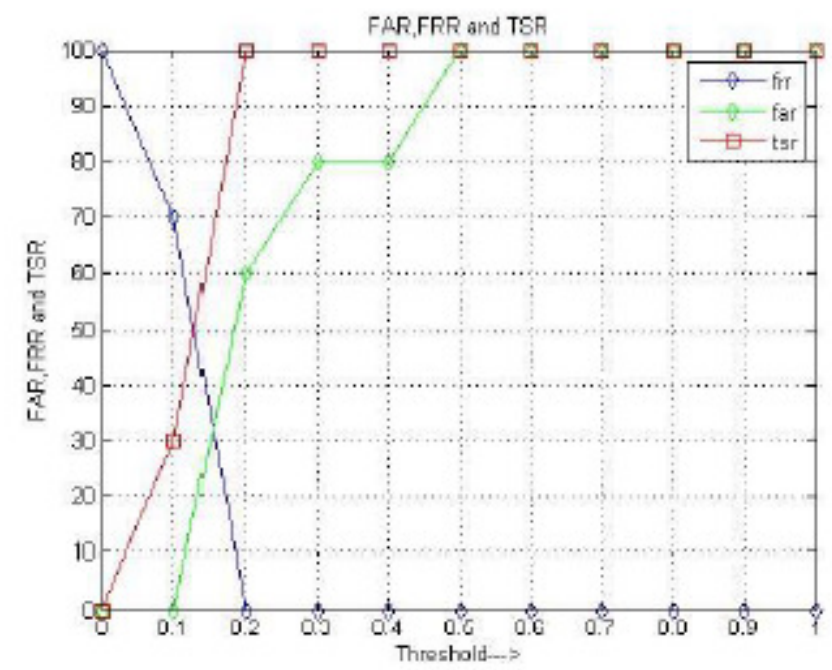

Figure 8. Performance parameters variations with threshold for YALE database

\subsubsection{Parameter variation with Indian male Face Database:-}

The face database is created by considering fifteen persons with six samples per person. ie., Ninety image samples in the database. The value of the FAR is computed by considering five persons as outside the database. The variations of FRR, FAR and TSR with threshold are shown in fig. 9

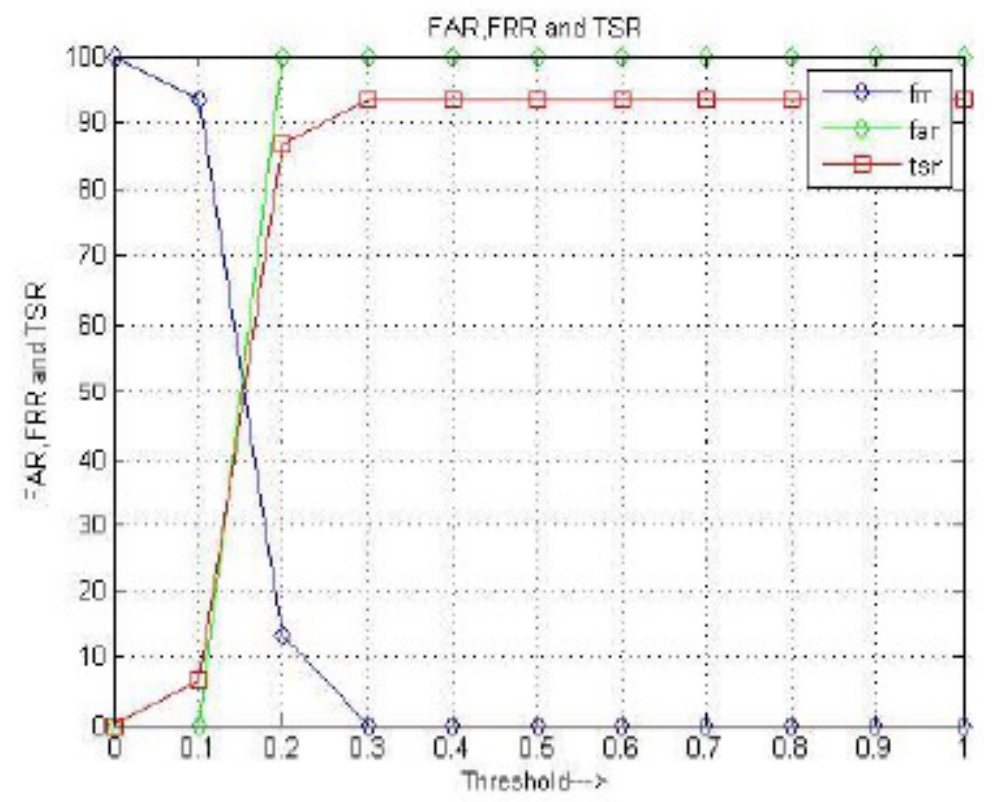

Figure 9. Performance parameters variations with threshold for Indian male database

The values of FAR and TSR increases with threshold values, where as the FRR decreases with threshold values. The value of EER is fifty and corresponding TSR value is fifty percent. 
Signal \& Image Processing : An International Journal (SIPIJ) Vol.7, No.2, April 2016

\subsubsection{Parameter variation with Face Database of Indian female:-}

The face database is formed by considering seventeen persons with six samples per person. ie., one hundred and two samples in the database. The value of the FAR is computed by considering five persons as outside the database. The variations of FRR, FAR and TSR with threshold are shown in fig. 10

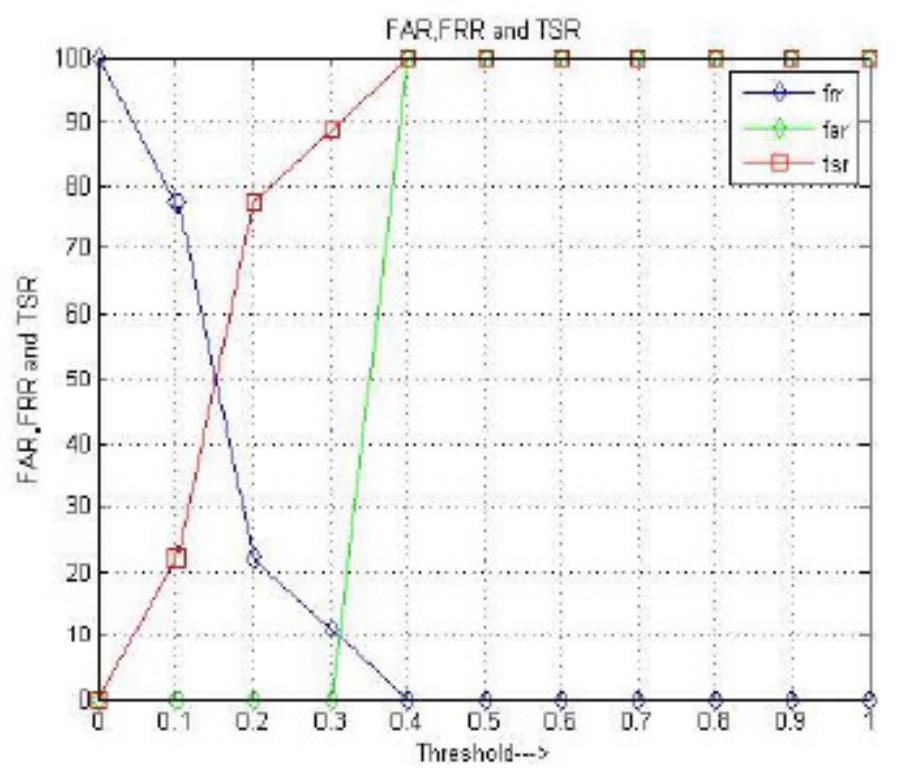

Figure 10. Performance parameters variations with threshold for Indian female database

The FAR and TSR increases with threshold values, where as the FRR decreases with threshold values. The value of EER is ten and corresponding TSR value is ninety one percent.

\subsection{Comparison of Performance parameters with different databases}

The performance parameters such as EER, Optimum TSR (opt. TSR) and Maximum TSR (Max. TSR) for different face databases viz., JAFFE, YALE, Indian Male and Indian Female are tabulated in table 2. The value of EER is low with higher values of opt. TSR and maximum TSR for JAFFE database. The performance of proposed algorithm is better with JAFFE database since variations in illumination and orientations are less. The performance of algorithm with Indian male database is very poor since the orientation in face images is very high.

Table 2: Comparison of EER, opt. TSR and Max. TSR for different face databases

\begin{tabular}{|c|c|c|c|}
\hline Databases & EER & Opt. TSR & Max.TSR \\
\hline JAFFE & 0 & 100 & 100 \\
\hline YALE & 32 & 70 & 100 \\
\hline Indian Male & 50 & 50 & 92.401 \\
\hline Indian Female & 10 & 91 & 100 \\
\hline
\end{tabular}


Signal \& Image Processing : An International Journal (SIPIJ) Vol.7, No.2, April 2016

\subsection{Comparison of proposed Algorithm with Existing Algorithms.}

The optimised percentage TSR of proposed method is compared with existing methods presented by Rangaswamy[23] and Ramesh and Raja[24] for JAFFE face database is given in table 3. It is noticed that the value of percentage TSR is better in the case of proposed method compared to existing methods.

Table 3: Comparison of proposed Algorithm with existing algorithms for JAFFE database

\begin{tabular}{|c|c|l|l|}
\hline S1.No & Authors & Techniques & TSR(\%) \\
\hline 1 & Rangaswamy et al.,[23] & DTCWT+FFT & 80 \\
\hline 2 & Ramesha K and K B Raja[24] & DTBFEFR & 90.3 \\
\hline 3 & Proposed method & $\begin{array}{l}\text { Log Transformation and } \\
\text { vector compression }\end{array}$ & 100 \\
\hline
\end{tabular}

The performance parameter values are better in the case of proposed method for the following reasons

i. The features are extracted using nonlinear technique.

ii. The single person's feature vectors of many samples of single person are fused to convert into single vector per person.

iii. The single feature vector of many samples of a person will optimize recognition rate.

iv. The method is useful for real time applications.

\section{CONCLUSiOnS}

The face recognition is powerful biometric trait to identify a person as the images can be captured without the knowledge of a person. In this paper, optimised face recognition system based on log transformation and combination of face image vectors is proposed. The face image quality is improved using Gaussian filter. The log transformation is used to extract features. The novel concept of converting many image feature vectors of single person into single feature vector using fusion technique to optimize the algorithm is introduced. It is observed that the performance parameters of the proposed algorithm are improved compared to existing algorithms. In future, algorithm can be tested with other biometric traits and can be combined for better performance. The algorithm can be tested for optimisation using hardware.

\section{REFERENCES}

[1] Abhijith Punnappurath and Ambasamudram Narayanan Rajagopalan "Face Recognition Across NonUniform Motion Blur, Illumination and Pose", IEEE Transactions on Image Processing, vol. 24, no. 7, pp. 2067-2082, July 2015.

[2] Yong Xu, Xiaozhao Fang, Xuelong Li, Jiang Yang, Jane You, Hong Liu and Shaohua Teng, "Data Uncertainty in Face Recognition", IEEE Transactions on Cybernetics, vol. 44, no. 10, pp. 1950-1961, October 2014

[3] Chia-Po Wei, Chih-Fan Chen, and Yu-Chiang Frank Wang, "Robust Face Recognition With Structurally Incoherent Low-Rank Matrix Decomposition", IEEE Transactions on Image Processing, Vol. 23, No. 8, pp. 3294-3307, August 2014.

[4] Muwei Jian and Kin-Man Lam, "Simultaneous Hallucination and Recognition of Low-Resolution Faces Based on Singular Value Decomposition", IEEE Transactions on Circuits and Systems for Video Technology, Vol. 25, No. 11, pp. 1761-1772, November 2015 
Signal \& Image Processing : An International Journal (SIPIJ) Vol.7, No.2, April 2016

[5] Changxing Ding, Jonghyun Choi, Dacheng Tao and Larry S. Davis, "Multi-Directional Multi-Level Dual-Cross Patterns for Robust Face Recognition", IEEE Transactions on Pattern Analysis and Machine Intelligence, vol. 38, issue 3, pp. 518 - 531, July 2015.

[6] Yiwen Wang, Lei Jiang,, Yun Wang Bangyu Cai,Yueming Wang, Weidong Chen, Sanyuan Zhang, and Xiaoxiang Zheng, "An Iterative Approach for EEG-Based Rapid Face Search: A Refined Retrieval by Brain Computer Interfaces", IEEE Transactions on Autonomous Mental Development, Vol. 7, No. 3, pp. 211-222, September 2015.

[7] Leyuan Fang and Shutao Li, "Face Recognition by Exploiting Local Gabor Features with Multitask Adaptive Sparse Representation", IEEE Transactions on Instrumentation and Measurement, Vol. 64, No. 10, pp. 2605-2615, October 2015,

[8] Jiwen Lu, Venice Erin Liong, Gang Wang and Pierre Moulin, "Joint Feature Learning for Face Recognition", IEEE Transactions on Information Forensics and Security, Vol. 10, No. 7, pp. 15711383, July 2015.

[9] Hamdi Dibeklio glu, Fares Alnajar, Albert Ali Salah and Theo Gevers, "Combining Facial Dynamics with Appearance for Age Estimation", IEEE Transactions on Image Processing, Vol. 24, No. 6, pp. 1928-1943, June 2015.

[10] B. Rosario Campomanes-Álvarez, Oscar Ibá̃nez, Carmen Campomanes-Álvarez, Sergio Damas, and Oscar Cordón, "Modeling Facial Soft Tissue Thickness for Automatic Skull-Face Overlay", IEEE Transactions on Information Forensics and Security, Vol. 10, No. 10, pp. 2057-2070, October 2015.

[11] Shenghua Gao, Yuting Zhang, Kui Jia, Jiwen Lu and Yingying Zhang, "Single Sample Face Recognition via Learning Deep Supervised Auto Encoders", IEEE Transactions on Information Forensics and Security, Vol. 10, No. 10, pp. 2108-2118, October 2015.

[12] Yiwen Wang, Lei Jiang, Yun Wang, Bangyu Cai,Yueming Wang, Weidong Chen, Sanyuan Zhang, and Xiaoxiang Zheng, "An Iterative Approach for EEG-Based Rapid Face Search: A Refined Retrieval by Brain Computer Interfaces", IEEE Transactions on Autonomous Mental Development, Vol. 7, No. 3, pp. 211-222, September 2015.

[13] Xiaochun Cao, Changqing Zhang and Chengju Zhou, "Huazhu Fu, and Hassan Foroosh, Constrained Multi-View Video Face Clustering", IEEE Transactions on Image Processing, Vol. 24, No. 11, pp. 4381-4393, November 2015.

[14] Xudong Jiang and Jian Lai, "Sparse and Dense Hybrid Representation via Dictionary Decomposition for Face Recognition", IEEE Transactions on Pattern Analysis and Machine Intelligence, Vol. 37, No. 5, pp. 1067-1079, May 2015.

[15] Priyanka V. Bankar and Anjali C. Pise, "Face Recognition by using GABOR and LBP", IEEE International Conference on Communications and Signal Processing (ICCSP 2015) pp. 45-48, April 2015.

[16] Muhammad Uzair, Arif Mahmood and Ajmal Mian. "Hyperspectral Face Recognition with Spatiospectral Information Fusion and PLS Regression", IEEE Transactions on Image Processing, Vol. 24, No. 3, pp. 1127-1137, March 2015.

[17] Samiksha and Pallavi Khatri, "Facial Expression Detection Techniques: based on Viola and Jones Algorithm and Principal Component Analysis", Fifth International Conference on Advanced Computing and Communication Technologies (ACCT), PP. 108-112, February 2015.

[18] Xiaoshuang Shi, Zhenhua Guo, Feiping Nie, Lin Yang, Jane You, and Dacheng Tao, "Two Dimensional Whitening Reconstruction for Enhancing Robustness of Principal Component Analysis", IEEE Transactions on Pattern Analysis and Machine Intelligence, issue 99, November 2015.

[19] http://www.kasrl.org/jaffe_download.html.

[20] http://vision.ucsd.edu/datasets/yale_face_dataset_original/yalefaces.zip

[21] http://viswww.cs.umass.edu/ vidit/IndianFaceDatabase

[22] Guruprasad G and Veena S K, "Face Recognition using Transform Domain Techniques", IOSR Journal of VLSI and Signal Processing (IOSR-JVSP) Volume 5, Issue 3, Ver. II, May - Jun. 2015, PP $06-12$ 
Signal \& Image Processing : An International Journal (SIPIJ) Vol.7, No.2, April 2016

[23] Y. Rangaswamy K B Raja and K R Venugopal, "Face Recognition using Fusion of DTCWT and FFT Features" Eleventh International Multi-Conference on Information Processing-2015 (IMCIP- 2015), pp. 809-817, 2015.

[24] Ramesha K and K B Raja, " Dual Transform based Feature Extraction for Face Recognition" International Journal of Computer Science Issues, Vol. 8, Issue 5, No 2, pp. 115-124, September 2011.

\section{AUTHORS}

Sateesh Kumar H.C. is a Associate Professor in the Dept of Electronics and Communication Engineering at Sai Vidya Institute of Technology, Bangalore. He obtained his B.E. degree in Electronics Engineering from Bangalore University. His specialization in Master degree was Bio-Medical Instrumentation from Mysore University and currently he is pursuing Ph.D. in the area of Image segmentation under the guidance of Dr. K B Raja, Professor, Dept of Electronics and Communication Engineering, University Visvesvaraya college of Engineering, Bangalore. He has over

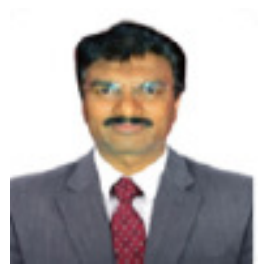
23 research publications in refereed International Journals and Conference Proceedings. His area of interest is in the field of Signal Processing and Communication Engineering. He is the life member of Institution of Engineers (India), Institution of Electronics and Telecommunication Engineers and Indian society for Technical Education.

Raja K.B. is a Professor, Department of Electronics and Communication Engineering, University Visvesvaraya college of Engineering, Bangalore University, Bangalore. He obtained his B.E and M.E in Electronics and Communication Engineering from University Visvesvaraya College of Engineering, Bangalore. He was awarded Ph.D. in Computer Science and Engineering from Bangalore University. He has over 180 research publications in refereed International Journals and Conference Proceedings. He has guided $11 \mathrm{Ph} . \mathrm{D}$ students. His research interests include Image Processing, Biometrics, VLSI Signal Processing, computer networks.

Venugopal K.R. is currently the Principal and Dean, Faculty of Engineering, University Visvesvaraya College of Engineering, Bangalore University, Bangalore. He obtained his Bachelor of Engineering from University Visvesvaraya College of Engineering. He received his Master's degree in Computer Science and Automation from Indian Institute of Science, Bangalore. He was awarded Ph.D. in Economics from Bangalore University and Ph.D. in Computer Science from Indian Institute of Technology, Madras. He has a distinguished academic career and has degrees in Electronics, Economics, Law, Business Finance, Public Relations, Communications,

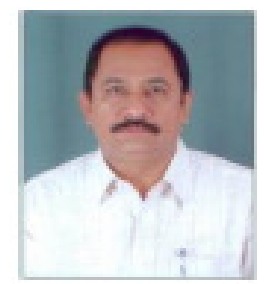
Industrial Relations, Computer Science and Journalism. He has authored 27 books on Computer Science and Economics, which include Petrodollar and the World Economy, C Aptitude, Mastering C, Microprocessor Programming, Mastering C++ etc. He has been serving as the Professor and Chairman, Department of Computer Science and Engineering, University Visvesvaraya College of Engineering, Bangalore University, Bangalore. During his three decades of service at UVCE he has over 520 research papers to his credit. His research interests include computer networks, parallel and distributed systems, digital signal processing and data mining. 